\title{
Evolutionary dynamics of olfactory receptor genes in chordates: Interaction between environments and genomic contents
}

\author{
Yoshihito Niimura* \\ Department of Bioinformatics, Medical Research Institute, Tokyo Medical and Dental University, Tokyo, Japan \\ *Correspondence to: Tel: +81 35803 4595; Fax: +81 35803 0247; E-mail: niimura@bioinfo.tmd.ac.jp
}

Date received (in revised form): 13th July 2009

\begin{abstract}
Olfaction is essential for the survival of animals. Versatile odour molecules in the environment are received by olfactory receptors (ORs), which form the largest multigene family in vertebrates. Identification of the entire repertories of OR genes using bioinformatics methods from the whole-genome sequences of diverse organisms revealed that the numbers of OR genes vary enormously, ranging from $\sim 1,200$ in rats and $\sim 400$ in humans to $\sim 150$ in zebrafish and $\sim 15$ in pufferfish. Most species have a considerable fraction of pseudogenes. Extensive phylogenetic analyses have suggested that the numbers of gene gains and losses are extremely large in the OR gene family, which is a striking example of the birth-and-death evolution. It appears that OR gene repertoires change dynamically, depending on each organism's living environment. For example, higher primates equipped with a well-developed vision system have lost a large number of OR genes. Moreover, two groups of OR genes for detecting airborne odorants greatly expanded after the time of terrestrial adaption in the tetrapod lineage, whereas fishes retain diverse repertoires of genes that were present in aquatic ancestral species. The origin of vertebrate OR genes can be traced back to the common ancestor of all chordate species, but insects, nematodes and echinoderms utilise distinctive families of chemoreceptors, suggesting that chemoreceptor genes have evolved many times independently in animal evolution.
\end{abstract}

Keywords: chordate, mammal, molecular evolution, multigene family, olfactory receptor, vertebrate

\section{Introduction}

There are a variety of odours in our environment. Detecting molecules of $\beta$-phenylethyl alcohol is recognised as the fragrance of rose in the human brain, while amyl acetate is perceived as a banana flavour. To humans, olfaction - the sense of smellmay seem to be less important than vision or hearing. To most animals, however, olfaction is essential for their survival. It is used to find foods, avoid predators and identify mates and offspring. Odour molecules in the environment are detected by olfactory receptors (ORs). OR genes were first identified from rats by Buck and Axel in 1991. ${ }^{1}$ They discovered a huge multigene family of G-protein-coupled receptors (GPCRs), of which expression is restricted to the olfactory epithelium in the nasal cavity, and suggested that there are $\sim 1,000$ different $\mathrm{OR}$ genes in mammalian genomes. The discovery opened the door to the molecular study of chemosensation, and these authors received the Nobel Prize in 2004 for this achievement. GPCRs are membrane proteins with 
seven transmembrane $\alpha$-helical regions. The binding of a ligand to a receptor activates a G-protein and a subsequent signalling cascade. GPCRs are classified into at least five groups by sequence similarities. ${ }^{2}$ OR genes belong to the largest group of them, the rhodopsin-like GPCR superfamily, which also includes opsin genes (which encode proteins that are photosensitive) and many other receptor genes, including those encoding neurotransmitters, peptide hormones, chemokines, lipids and nucleotides. OR genes are intronless in their coding regions, although the number of exons in the $5^{\prime}$ untranslated region can be variable and these non-coding exons are often alternatively spliced. ${ }^{3}$

It is generally thought that the olfactory system uses combinatorial coding. ${ }^{4}$ The relationships between odour molecules and $\mathrm{ORs}$ are not one-to-one, but multiple-to-multiple; that is, one OR recognises multiple odorants, and one odorant is recognised by multiple ORs. Therefore, different odorants are recognised by different combinations of ORs. This system would explain why tens of thousands of different odours can be detected and discriminated by using $\sim 1,000$ OR genes. It also explains why the classification of odours has long been unsuccessful. In the case of colours, we know that there are three primary colours (red, green and blue). The presence of the three primary colours can be explained by the fact that the human genome has three opsin genes, corresponding to 'red', 'green' and 'blue'. The three opsins are contained in different cone pigments, with sensitivities to radiation of different wavelengths. There are five primary tastes: sweet, bitter, salty, sour and umami. The last mentioned means 'deliciousness' in Japanese and is the taste of L-glutamate. It is known that each of these five tastes is detected by different genes or gene families. ${ }^{5,6}$ In the case of olfaction, however, there is no generally accepted classification of odours, regardless of long-standing efforts to identify 'primary odours' from which all other odours could be reproduced using appropriate mixtures. ${ }^{7}$ For example, Amoore proposed that the following are primary odours, on the basis of stereochemical properties of molecules: ethereal, camphoraceous, musky, floral, pepperminty, pungent and putrid. $^{8}$ The multiple-to-multiple relationships between odours and receptors, however, suggest that primary odours actually do not exist.

Because OR genes form the largest multigene family and comprise up to $4-5$ per cent of the entire proteome in mammals, they are interesting targets for the study of molecular evolution. By investigating the evolution of the OR gene family, it is expected that insights will be obtained not only into the biology of chemosensation, but also into the mechanisms of evolution of multigene families in general. Moreover, since olfaction is a sensor to the external world, studying the evolution of OR genes might tell us how the genomic contents interact with different environments. These days, the whole-genome sequences of diverse organisms have become available. Therefore, it is now possible to identify a nearly complete repertoire of OR genes encoded in each organism's genome. In this paper, recent progress on the evolution of OR genes is reviewed from the viewpoint of comparative genomics, by looking across a broad range of animal phylogeny.

\section{OR genes in humans}

The entire set of OR genes present in the human genome was determined by several groups. ${ }^{9-11} \mathrm{By}$ conducting extensive homology searches, $\sim 800$ OR genes were identified, but, interestingly, more than half of them were found to be pseudogenes (Figure 1). ${ }^{11}$ Therefore, the number of functional genes in humans is $<400$. Here, the distinction between a functional gene and a pseudogene was made on the basis of its sequence. When an intact coding sequence could be recovered with an initiation codon and a stop codon in the proper positions, such a sequence was considered to be functional, while a sequence disrupted by nonsense or frameshift mutations or long deletions was regarded as a pseudogene. OR genes form many genomic clusters and are scattered all over the human genome, with the exceptions of chromosome 20 and the $\mathrm{Y}$ chromosome. ${ }^{11}$ In particular, 


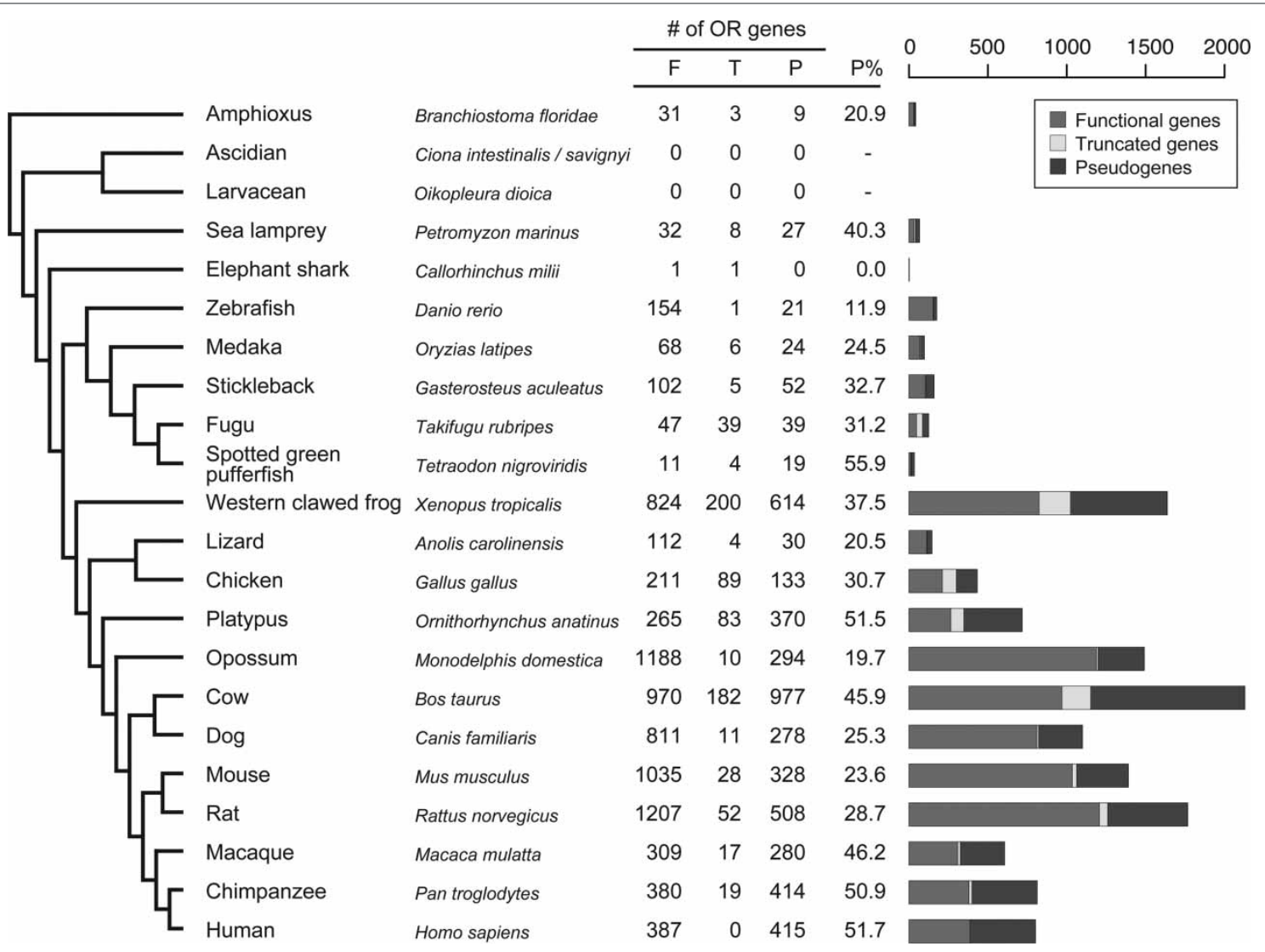

Figure I. Numbers of OR genes in 23 chordate species. ${ }^{12-14} \mathrm{~F}$, T and $\mathrm{P}$ indicate the numbers of functional genes, truncated genes and pseudogenes, respectively. A truncated gene is part of an intact sequence that is located at a contig end. The fraction of pseudogenes ( $P$ per cent) in each species was estimated by assuming that all truncated genes are functional. ${ }^{12}$ Phylogenetic relationships among the 23 species are also shown.

chromosome 11 contains $>40$ per cent of all OR genes. The genes located close to each other on a chromosome tend to be evolutionarily closely related, suggesting that the number of OR genes has increased by repeated tandem gene duplications. ${ }^{11}$ The relationships between genomic clusters and evolutionary kinships, however, are often complicated by chromosomal rearrangements. ${ }^{11}$ For example, it is known that human chromosomes 14 and 15 were generated by chromosome fission in the common ancestor of great apes, and that the fission event occurred at a cluster of OR genes. ${ }^{15}$ Nevertheless, the organisation of OR gene clusters are generally well conserved between humans and mice, and orthologous relationships can easily be identified between the two species. ${ }^{16}$ The number of OR genes in mice $(\sim 1,000$; Figure 1$)$ is much higher than that in humans, and thus each genomic cluster in mice contains a larger number of OR genes than in humans, on average. This observation suggests that the greater OR gene repertoires in mice relative to humans have been generated mainly by gene duplications within each cluster. ${ }^{16}$

Each OR is thought to be specialised to recognise physicochemical features of odour molecules, such as functional groups or molecular size; however, the relationships between ORs and odour molecules are largely unknown. Recently, Saito et al. ${ }^{17}$ conducted high-throughput screening of 93 different odours against 464 ORs expressed in heterologous cells and succeeded in identifying ligands for 52 mouse and ten human ORs. Yet, so far, ligands have been identified for only $\sim 100$ mammalian ORs. Mammalian OR genes are known to 
be classified into two groups, class I and class II, according to sequence similarity. ${ }^{18}$ The functional difference between class I and class II genes is still unclear, but it has been hypothesised that class I and class II genes are for detecting relatively hydrophilic and hydrophobic compounds, respectively. ${ }^{19}$

Human olfactory perception differs largely among individuals. For example, one individual in ten cannot perceive hydrogen cyanide, an extreme poisonous gas. ${ }^{20}$ One in 1,000 does not smell butyl mercaptan, the odour of the skunk. Such phenomena are called specific anosmia, meaning specific loss or impairment of smell. ${ }^{20}$ Another example is androstenone, a pig pheromone. This odour is perceived as offensive ('sweaty, urinous'), pleasant ('sweet, floral'), or odourless. Recently, Keller et al. ${ }^{21}$ revealed that the genetic variation in a human OR gene, OR7D4, which is selectively activated by androstenone, accounts for the variation in the perception of androstenone. There is a common variant of this receptor containing two amino acid substitutions, and homozygous or heterozygous subjects with these amino acid changes are less sensitive and have less unpleasant perceptions of androstenone.

It is also known that OR genes are highly polymorphic in humans. It has been reported that $>60$ OR loci are segregating pseudogenes, in which both an intact allele and a pseudogenised allele exist in the human population. ${ }^{22}$ Menashe et al. ${ }^{23}$ conducted a genome-wide association study and found a significant association between the presence of a nonsense single nucleotide polymorphism (SNP) in an OR, OR11H7P, and detection threshold differences for the sweaty odorant, isovaleric acid. Nozawa et al. ${ }^{24}$ examined copy number variations in human OR genes and suggested that the difference in the number of functional $\mathrm{OR}$ genes between two individuals is $\sim 11$, on average.

\section{OR genes in primates}

As shown in Figure 1, the number of OR genes is quite variable among different species. Figure 1 indicates that primates (humans, chimpanzees and macaques) have much smaller numbers (300-400) of functional OR genes than other mammals $(\sim 1,000) .{ }^{12,13}$ Moreover, the fractions of pseudogenes in primates $(\sim 50$ per cent) are much higher than those in rodents (mice and rats), dogs and opossums $(<30$ per cent). These observations are not unexpected, because it is generally thought that higher primates are vision-orientated animals and that their olfactory ability has retrogressed. In fact, it is known that the olfactory epithelium and the olfactory bulb, a brain region processing olfactory information, in primates are proportionately smaller than those in most other mammals. ${ }^{25}$

As explained earlier, human colour vision is mediated by three distinctive visual pigments. The blue-sensitive pigment gene is on chromosome 7 , while the red and green pigment genes lie adjacent to each other on the $\mathrm{X}$ chromosome. It is known that the gene duplication giving rise to the red and green pigment genes occurred in the common ancestor between hominoids (humans and apes) and old world monkeys (OWMs), because all of these primate species are trichromats, having three pigment genes. By contrast, most non-primate mammals and prosimians (primitive primates) are dichromatic - that is, colour-blind. The colour vision system in new world monkeys (NWMs) is complicated. Most NWMs have one pigment gene locus on the $\mathrm{X}$ chromosome, but the gene is polymorphic; therefore, heterozygous females can possess trichromatic vision, but all males and homozygous females are dichromatic.

To investigate the interaction between olfaction and colour vision, Gilad et al. ${ }^{26,27}$ estimated the fractions of OR pseudogenes in 19 primate species by sequencing 100 randomly chosen OR genes. They found that hominoids and OWMs show a significantly higher fraction of OR pseudogenes than NWMs and prosimians. This result may suggest that the acquisition of trichromatic vision caused the decline of olfactory ability in primates. However, Hiramatsu et al. ${ }^{28}$ reported that there is no significant difference between dichromats and trichromats in the foraging efficiency among wild spider monkeys in Costa Rica. Therefore, other factors may also need to be considered as the cause of olfactory retrogression in higher primates. 
Several studies proposed that the extent of OR gene loss is more prominent in humans than in chimpanzees. ${ }^{26,29,30}$ Our recent analyses using high-quality chimpanzee genome sequences, however, showed that the numbers of functional OR genes, the fractions of pseudogenes and the extents of pseudogenisation are very similar between humans and chimpanzees. ${ }^{13}$ At the same time, however, we found that the OR gene repertoires are different between the two species, despite their close evolutionary relationship: $\sim 25$ per cent of their functional OR gene repertories are species specific. It is therefore possible that the spectrum of detectable odorants might be different between the two species. We also estimated the numbers of functional OR genes and pseudogenes in the most recent common ancestor (MRCA) between humans and chimpanzees. ${ }^{13}$ The analysis showed that the MRCA had a larger number of functional OR genes $(>500)$ and a smaller fraction of pseudogenes (41 per cent) than humans and chimpanzees, supporting the idea that OR gene repertoires are in the process of degeneration in both human and chimpanzee lineages (Figure 2a).

\section{OR genes in mammals}

Figure 1 indicates that platypuses, as well as primates, have a smaller number of functional OR genes and a larger fraction of pseudogenes than other mammals. The platypus is an egg-laying mammal and belongs to the monotremes, the lineage of which diverged earliest among extant mammals. The observations of the small number of OR genes and the higher fraction of pseudogenes in the platypus may be explained in the following way. ${ }^{12}$ First, platypuses are semi-aquatic. Mammalian OR genes have been adapted to detecting airborne odorants, and thus they are apparently useless in the water (see below). Second, platypuses have a bill sense. The platypus's bill functions as both an electrical and mechanical sensor; they can find prey with their eyes, ears and nostrils closed in the mud at the bottom of streams. This situation is reminiscent of toothed whales (dolphins), which have completely lost the olfactory apparatus and instead have developed the echolocation system to adapt to the full aquatic life. In fact, recent studies have suggested that the fraction of OR pseudogenes in toothed whales is extremely high ( $>75$ per cent). ${ }^{31,32}$ By contrast, baleen whales, which have a significantly reduced but intact olfactory system, appear to have a lower fraction of OR pseudogenes than toothed whales. The relationship between the number of OR genes and the olfactory prowess of a species, however, is not always clear. For example, dogs are supposed to have a keen sense of smell, but their OR gene repertoire is not particularly large (Figure 1).

The evolutionary changes in the number of OR genes in mammals have been investigated. ${ }^{12}$ The results indicated that hundreds of gains and losses of OR genes have occurred in each branch (Figure 2b). This is consistent with the observation that every species examined has a large number of pseudogenes (Figure 1). Moreover, this finding suggests that, although the current numbers of functional $\mathrm{OR}$ genes in several mammalian species are similar $(\sim 1,000)$, their $\mathrm{OR}$ gene repertoires have been highly diversified. It appears that the OR gene family is characterised by dynamic changes in number during evolution, providing an extreme case of birth-and-death evolution of a multigene family. ${ }^{33}$

\section{OR genes in vertebrates}

OR genes are present in all vertebrate species. Figure 1 shows the numbers of OR genes in 14 non-mammalian species for which the draft genome sequences are available. ${ }^{14}$ Birds were thought to lack a well-developed sense of smell; however, behavioural studies have shown that some bird species use olfaction to navigate, forage and distinguish individuals. A recent study, using nine diverse bird species, estimated that they have 100700 OR genes, including pseudogenes, in their genomes. ${ }^{34}$ Western clawed frogs have a surprisingly large repertoire of OR genes, which is comparable to that in most mammals. ${ }^{14}$ Fish detect mainly four groups of water-soluble molecules as odorants: amino acids, gonadal steroids, bile acids 


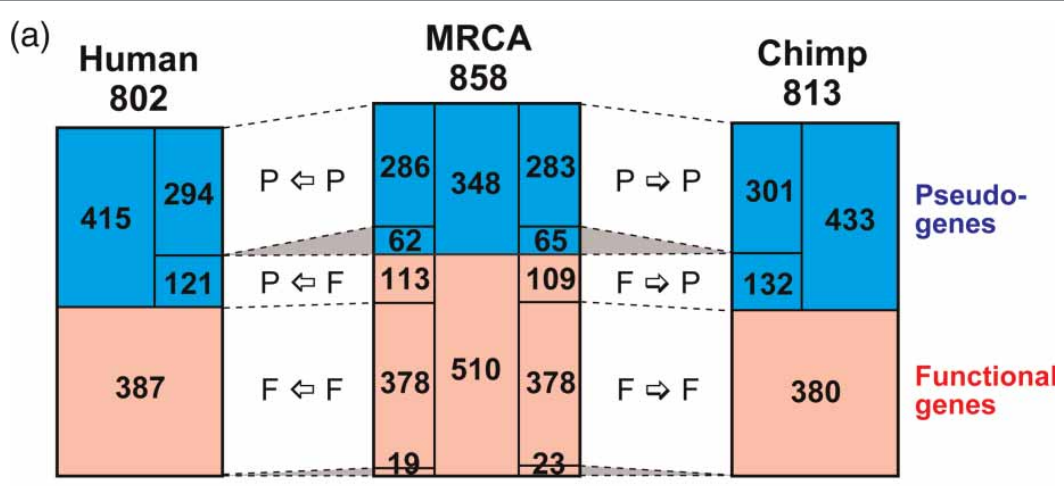

(b)

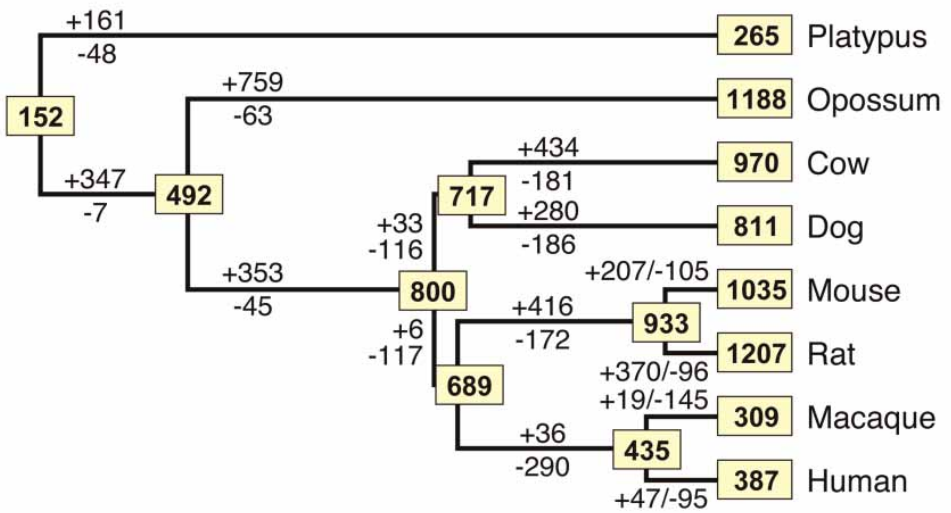

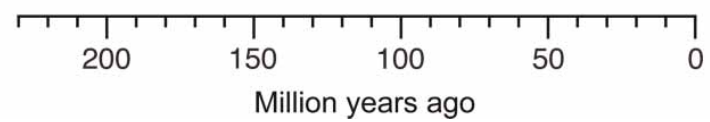

Figure 2. Evolutionary changes in the number of olfactory receptor (OR) genes in mammals. (a) Estimated numbers of functional OR genes (red) and OR pseudogenes (blue) in the most recent common ancestor (MRCA) between humans and chimpanzees. The numbers in humans and chimpanzees are also shown. ' $F \rightarrow F$ ' ('P $\rightarrow$ ') indicates that a functional gene (a pseudogene) in the MRCA is still functional (a pseudogene) in humans or chimpanzees. ' $\mathrm{F} \rightarrow \mathrm{P}$ ' represents a functional gene in the MRCA becoming a pseudogene in the human or chimpanzee lineage. A grey triangular area depicts deletion from the genome; for example, 62 pseudogenes in the MRCA were eliminated from the human genome. Adapted from Go and Niimura (2008). ${ }^{13}$ (b) Gains and losses of OR genes in mammalian evolution. A number in a box represents the number of functional OR genes in the extant or ancestral species. A number with a plus and a minus sign indicates the number of gene gains and losses, respectively, for each branch. Adapted from Niimura and Nei (2007). ${ }^{12}$

and prostaglandins. These odorants are non-volatile, so humans cannot smell them. As shown in Figure 1, teleost fish (zebrafish, medaka, stickleback, fugu and spotted green pufferfish) generally have much smaller numbers of OR genes than mammals, but the number of functional OR genes varies considerably among species, as was observed in mammals.

Sea lampreys and elephant sharks-a jawless vertebrate and a cartilaginous fish, respectively-are two early-diverging lineages in vertebrates (see Figure 1). Sea lampreys have a comparable number of OR genes to teleost fish (Figure 1). It is known that sea lampreys possess a well-developed olfactory system and have a relatively large olfactory bulb. ${ }^{35}$ They are anadromous and migrate to shallow water streams for spawning utilising odour cues. By contrast, only one intact OR gene and one truncated gene were identified from the elephant shark genome. Although the coverage of the elephant shark genome is low $(1.4 \times),{ }^{36}$ the number of OR genes in this species appears to be surprisingly small. Sharks are famous for their remarkably acute sense of smell; however, elephant sharks are 
distantly related to sharks and rays. They live in the deep sea ( $\sim 200$ metres), and their ecology is not yet well understood.

Extensive phylogenetic analyses showed that vertebrate OR genes can be separated into type 1 and type 2 genes (Figure 3a). ${ }^{14,37}$ As mentioned earlier, mammalian OR genes are classified into class I and class II, but both classes belong to type 1.,37 Figure $3 \mathrm{a}$ indicates that fish $\mathrm{OR}$ genes are more diverse than mammalian OR genes, although the number of OR genes in fish is much smaller than in mammals. Both type 1 and type 2 clades contain lamprey OR genes, suggesting that the divergence between type 1 and type 2 clades was more ancient than the divergence between jawless and jawed vertebrates. OR genes in teleost fish and tetrapods (amphibians, reptiles, birds and mammals) were classified into seven groups, named $\alpha-\eta$, each of which corresponds to at least one ancestral gene in the MRCA between teleost fish and tetrapods. ${ }^{14,37}$ Among the seven groups, $\alpha-\zeta$ belong to type 1 , while group $\eta$ is included in the type 2 clade. The type 2 clade contains some diverse genes other than group $\eta$ genes, but they are apparently non-OR genes. ${ }^{14}$ Figure $3 \mathrm{~b}$ shows the numbers of OR genes belonging to groups $\alpha-\zeta$ in each species. Interestingly, group $\alpha$ and $\gamma$ genes are present in tetrapods but are absent in fish, with the exception of one intact gene in zebrafish. By contrast, group $\delta, \varepsilon, \zeta$ and $\eta$ genes are found in teleost fish and amphibians, whereas reptiles, birds and mammals completely lack them. From this observation, it is suggested that the former groups of genes are for detecting airborne odorants, while the latter groups are for water-soluble odorants. ${ }^{14,37}$ Group $\beta$ genes, however, were found to be present both in aquatic and terrestrial vertebrates. It is therefore possible to speculate that group $\beta$ genes detect odorants that are both water soluble and airborne. ${ }^{14}$ In mammals, group $\gamma$ corresponds to class II, whereas groups $\alpha$ and $\beta$ correspond to class I.

The evolutionary scenario of vertebrate OR genes was inferred in the following way. ${ }^{37}$ The MRCA of teleost fish and tetrapods had a diverse set of OR genes for water-soluble odorants (Figure 4). In the tetrapod lineage, two groups of genes ( $\alpha$ and $\gamma$ ) acquired the ability to detect airborne odorants, and these groups of genes expanded enormously, probably because the importance of olfaction is larger in terrestrial organisms than in marine organisms. In reptiles, birds and mammals, the genes specific to water-soluble odorants have been eliminated from the genome, because they are useless in terrestrial life. By contrast, amphibians retain the genes for both water-soluble and airborne odorants, reflecting their adaptation to both aquatic and terrestrial environments.

\section{OR genes in invertebrates}

Humans belong to the phylum Chordata. Chordates include cephalochordates, urochordates and vertebrates. Amphioxus is a cephalochordate, and ascidians and larvaceans are urochordates (see Figure 1). Amphioxus is called an 'acraniate', meaning a headless organism, and it lacks any distinctive olfactory organs. Nevertheless, examination of the amphioxus genome sequences revealed that the amphioxus has $>30$ vertebrate-type $\mathrm{OR}$ genes (Figure 1). ${ }^{14}$ Phylogenetic analyses showed that these amphioxus genes formed a monophyletic clade with all vertebrate OR genes and were clearly distinguishable from other non-OR GPCR genes. The amphioxus genes form an amphioxus-specific clade, suggesting that gene expansion has occurred in the amphioxus lineage (Figure 3a). Satoh ${ }^{38}$ reported that at least one OR gene is expressed in bipolar neurones embedded within the rostral epithelium of adult amphioxus, but further studies are necessary to examine the chemosensory system of the amphioxus. Recent phylogenomic analyses demonstrated that cephalochordates, rather than urochordates, are the most basal lineage in chordates. ${ }^{39,40}$ Therefore, the origin of the vertebratetype OR gene family can now be traced back to the common ancestor of all chordates species (Figure 4). ${ }^{14}$

By contrast, no OR-like genes were found from the genome sequences of three urochordate species (Figure 1). The absence of vertebrate-type OR genes in the urochordate genomes examined suggests that all OR genes were lost in their lineages 
(a)

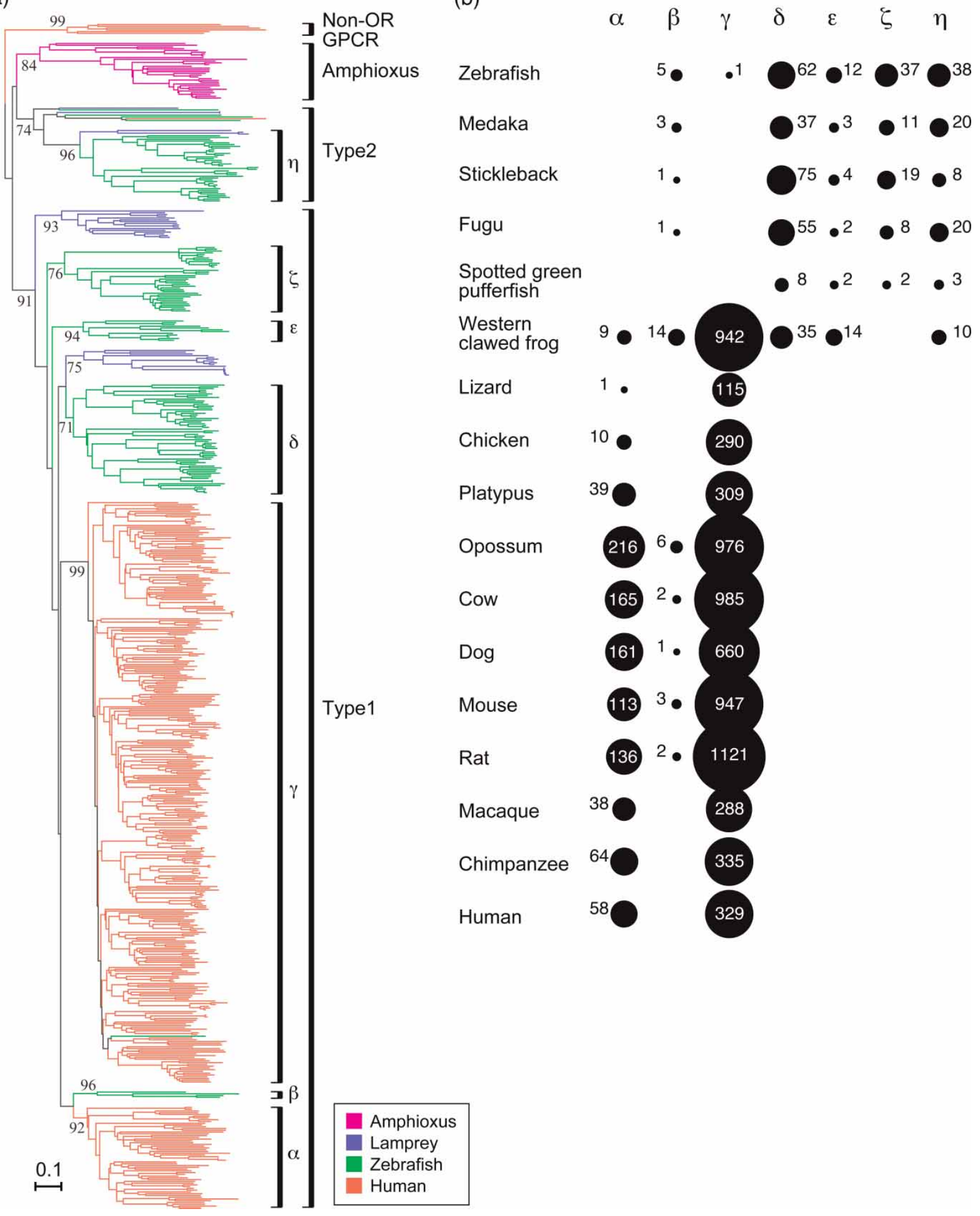

(b)

$\begin{array}{lllllll}\alpha & \beta & \gamma & \delta & \varepsilon & \zeta & \eta\end{array}$

Stickleback $\quad 1 \bullet \quad 0^{75} \bullet^{4} \bullet^{19}$

Fugu $\quad 1 \bullet \quad \bullet^{55} \bullet^{8} \bigcirc^{20}$

Spotted green

pufferfish

Western

clawed frog

Lizard

Chicken $\quad{ }^{10}$

Platypus $\quad 390$

Opossum $216{ }^{6}$

Cow

Dog

Mouse

Rat

Macaque

Chimpanzee

Human

329

Figure 3. (a) Neighbour-joining phylogenetic tree containing all functional olfactory receptors (OR) genes in the amphioxus, lamprey, zebrafish and human. Non-OR G-protein coupled receptor (GPCR) genes were used as the outgroup. The Type 2 clade contains several non-OR genes that are not included in group $\eta .{ }^{14}$ Bootstrap values obtained from 500 resamplings are shown for major clades. A scale bar represents the estimated number of amino acid substitutions per site. Adapted from Niimura (2009). ${ }^{14}$ (b) Numbers of functional OR genes (including truncated genes) belonging to groups $\alpha-\eta$ in teleost fish and tetrapods. ${ }^{14}$ 


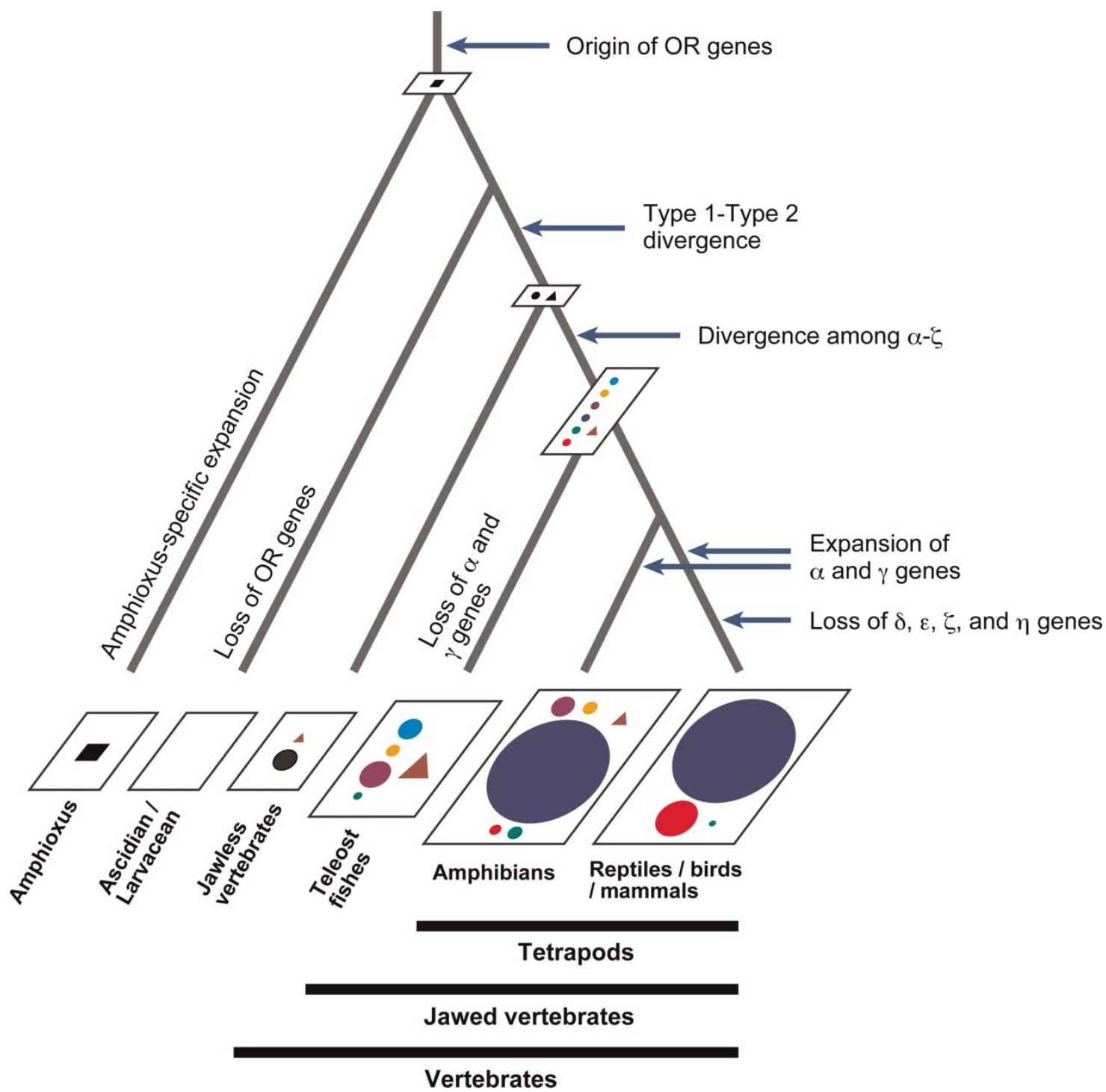

Figure 4. The evolutionary scenario of OR genes in chordates. ${ }^{14,37}$ Phylogenetic analyses suggested that the divergence between type I (circles) and type 2 (triangles) occurred before the divergence between jawless and jawed vertebrates, and the divergence among groups $\alpha-\zeta$ (circles in different colours) predate the divergence between teleost fish and tetrapods. In terrestrial organisms, group $\alpha$ (red circles) and $\gamma$ genes (blue circles) have largely expanded.

(Figure 4). Ascidians are sessile filter feeders, while larvaceans have a floating planktonic lifestyle. Reflecting their relatively inactive lifestyles, the nervous systems of urochordates are highly reduced and sensory receptors are poorly developed. The possibility that other families of unannotated genes act as chemosensory receptors in urochordates cannot, however, be excluded (see below).

Outside the phylum Chordata, no vertebratetype OR genes have so far been identified. The sea urchin belongs to the phylum Echinodermata, which is a close relative of the phylum Chordata. Raible et al. ${ }^{41}$ reported that two large families of rhodopsin-like GPCR genes, which are specifically expanded in the sea urchin lineage and are distinct from vertebrate-type OR genes, putatively function as chemosensory receptors in the sea urchin. These genes are expressed in pedicellariae and the tube feet of adult sea urchins, structures that react to chemical stimuli. 
Insects also have genes called ORs (or odorant receptors). $6,42,43$ The fruit fly and the malaria mosquito have 62 and 79 odorant receptors genes, respectively. In flies, the olfactory organs are the antenna and the maxillary palp on the head. There are common features in neuroanatomy between the insect and vertebrate olfactory systems, but insect and vertebrate $\mathrm{OR}$ genes are strikingly different and share no sequence similarity. Insect ORs have seven transmembrane regions, but their membrane topology is inverted compared with that of classical GPCRs. ${ }^{44}$ Moreover, insect odorant receptors always function as heterodimers. A divergent member of the odorant receptor gene family heterodimerises with the ubiquitously expressed receptor, Or83b, forming an active receptor complex. ${ }^{45}$ Recently, it has been revealed that an insect odorant receptor complex is a ligand-gated ion channel, rather than a GPCR. ${ }^{46,47}$

The nematode Caenorhabditis elegans has a highly developed chemosensory system. The C. elegans genome apparently encodes $\sim 1,300$ potentially functional chemoreceptors and $\sim 400$ pseudogenessurprisingly large for this small animal, which consists of only $\sim 1,000$ somatic cells. $^{42,48}$ This large genetic investment may be due to an extreme dependence on chemosensory abilities in the absence of visual and auditory systems. Nematode chemoreceptors are GPCRs and are classified into many distinctive families. They have complex gene structures with one to eight introns, and the intron locations are different among families. Recently, Cummins et al. ${ }^{49}$ reported a novel family of putative chemoreceptor genes in the marine mollusc Aplysia californica. These genes are also rhodopsin-like GPCR genes, and $\sim 90$ chemoreceptor genes were identified from the lowcoverage $(2 \times) A$. californica genome.

It is often said that olfaction is the most 'primitive' sense in organisms. Even bacteria detect chemical molecules in the environment. They swim towards higher concentrations of attractants, such as sugars or amino acids, and away from repellents of toxic substances. As explained above, however, olfactory receptor (chemoreceptor) genes are different among bacteria, nematodes, molluscs, insects, echinoderms and chordates, although the chemosensory system is present in essentially all motile organisms. Therefore, it appears that genes involved in olfaction have evolved many times, independently, in each lineage. The chordate OR gene family would have emerged from one of the rhodopsin-like GPCR genes that was present in the ancestral bilaterian species.

\section{Future directions}

As this review has described, the OR multigene family has been changing dynamically during evolution. The number of OR genes in each lineage increases or decreases in response to the external world. For example, if an organism acquires a welldeveloped visual sense, a large number of OR genes will become pseudogenes and ultimately be eliminated from the genome. Genome-sequencing projects are currently ongoing for diverse organisms. The availability of new genome sequences will provide us with further knowledge on the interaction between environments and genomic contents. It should be noted that the relationship between the changes in OR gene number and environmental factors is not always clear, however, such as in the case of dogs or frogs. Currently, a major obstacle is the limited knowledge about which odorants bind to which OR, although a considerable amount of ligand-receptor information has been obtained as a result of novel experimental techniques. ${ }^{17}$ Deciphering the olfactory coding will greatly enhance our understanding of the evolutionary dynamics of this tremendous gene family.

Another dimension of this research is to make a comparison with other gene families. For example, a comparison of evolutionary dynamics between vertebrate and insect $\mathrm{OR}$ genes suggested that gains and losses of genes are less frequent in insect OR genes than in vertebrate OR genes. ${ }^{22,50}$ It is known that vertebrates have five further multigene families that are involved in chemosensation: trace amine-associated receptors, vomeronasal receptors types 1 and 2 for olfactory and pheromone perception, and taste receptors types 1 and 2 for taste perception (see Niimura and $\mathrm{Nei}^{5}$ and Nei et al. ${ }^{6}$ and the references therein). Very recently, a family of 
formyl peptide receptor-like protein was also found to have olfactory function. ${ }^{51,52}$ Among these gene families, the OR gene family is by far the largest. The evolutionary dynamics of these families have also been studied extensively. ${ }^{53-56}$ These studies suggest that the evolutionary patterns differ considerably among different gene families. For example, taste receptor genes appear to be evolutionarily more stable than OR genes, ${ }^{6}$ while vomeronasal receptor genes have changed more drastically in evolution than OR genes. ${ }^{54,57}$

The evolution of opsin genes is in sharp contrast to that of OR genes. Opsin genes also belong to the rhodopsin-like GPCR superfamily, and thus they are distant relatives of OR genes. Homologous opsin genes can be identified in vertebrates, insects and other invertebrates, however, indicating that the origin of the opsin gene family is much more ancient than that of the OR gene family. ${ }^{58}$ It is clear that several distinct opsin genes already existed in the common ancestor of bilaterians. Comparative analyses with various multigene families, together with the integration of sequence and functional data, will enable us to elucidate the commonality and uniqueness of evolutionary mechanisms of the OR gene family.

\section{Acknowledgments}

I thank Takeshi Hase, Hiromi Matsumae, So Nakagawa and Soichi Ogishima for helpful comments. This work was supported in part by a grant (20770192) from the Ministry of Education, Culture, Sports, Science and Technology, Japan.

\section{References}

1. Buck, L. and Axel, R. (1991), 'A novel multigene family may encode odorant receptors: A molecular basis for odor recognition', Cell Vol. 65, pp. $175-187$.

2. Fredriksson, R., Lagerström, M.C., Lundin, L.G. and Schiöth, H.B. (2003), 'The G-protein-coupled receptors in the human genome form five main families. Phylogenetic analysis, paralogon groups, and fingerprints', Mol. Pharmacol. Vol. 63, pp. 1256-1272.

3. Young, J.M., Shykind, B.M., Lane, R.P., Tonnes-Priddy, L. et al. (2003), 'Odorant receptor expressed sequence tags demonstrate olfactory expression of over 400 genes, extensive alternate splicing and unequal expression levels', Genome Biol. Vol. 4, p. R71.

4. Malnic, B., Hirono, J., Sato, T. and Buck, L.B. (1999), 'Combinatorial receptor codes for odors', Cell Vol. 96, pp. 713-723.

5. Niimura, Y. and Nei, M. (2006), 'Evolutionary dynamics of olfactory and other chemosensory receptor genes in vertebrates', J. Hum. Genet. Vol. 51 , pp. $505-517$.
6. Nei, M., Niimura, Y. and Nozawa, M. (2008), 'The evolution of animal chemosensory receptor gene repertoires: Roles of chance and necessity', Nat. Rev. Genet. Vol. 9, pp. 951-963.

7. Amoore, J.E. (1977), 'Specific anosmia and the concept of primary odors', Chem. Senses Vol. 2, pp. 267-281.

8. Amoore, J.E. (1963), 'Stereochemical theory of olfaction', Nature Vol. 198, pp. $271-272$.

9. Glusman, G., Yanai, I., Rubin, I. and Lancet, D. (2001), 'The complete human olfactory subgenome', Genome Res. Vol. 11, pp. 685-702.

10. Zozulya, S., Echeverri, F. and Nguyen, T. (2001), 'The human olfactory receptor repertoire', Genome Biol. Vol. 2, pp. research0018.1-0018.12.

11. Niimura, Y. and Nei, M. (2003), 'Evolution of olfactory receptor genes in the human genome', Proc. Natl. Acad. Sci. USA Vol. 100, pp. 12235-12240.

12. Niimura, Y. and Nei, M. (2007), 'Extensive gains and losses of olfactory receptor genes in mammalian evolution', PLoS ONE Vol. 2, p. e708.

13. Go, Y. and Niimura, Y. (2008), 'Similar numbers but different repertoires of olfactory receptor genes in humans and chimpanzees', Mol. Biol. Evol. Vol. 25, pp. 1897-1907

14. Niimura, Y. (2009), 'On the origin and evolution of vertebrate olfactory receptor genes: Comparative genome analysis among 23 chordate species', Genome Biol. Evol. Vol. 1, pp. 34-44.

15. Rudd, M.K., Endicott, R.M., Friedman, C., Walker, M. et al. (2009), 'Comparative sequence analysis of primate subtelomeres originating from a chromosome fission event', Genome Res. Vol. 19, pp. 33-41.

16. Niimura, Y. and Nei, M. (2005), 'Comparative evolutionary analysis of olfactory receptor gene clusters between humans and mice', Gene Vol. 346, pp. 13-21.

17. Saito, H., Chi, Q., Zhuang, H., Matsunami, H. and Mainland, J.D. (2009), 'Odor coding by a mammalian receptor repertoire', Sci. Signal. Vol. 2, ra9.

18. Glusman, G., Bahar, A., Sharon, D., Pilpel, Y. et al. (2000), 'The olfactory receptor gene superfamily: Data mining, classification, and nomenclature', Mamm. Genome Vol. 11, pp. 1016-1023.

19. Zhang, X. and Firestein, S. (2002), 'The olfactory receptor gene superfamily of the mouse', Nature Neurosci. Vol. 5, pp. 124-133.

20. Amoore, J.E. (1967), 'Specific anosmia: A clue to the olfactory code', Nature Vol. 214, pp. 1095-1098.

21. Keller, A., Zhuang, H., Chi, Q., Vosshall, L.B. et al. (2007), 'Genetic variation in a human odorant receptor alters odour perception', Nature Vol. 449, pp. 468-472.

22. Menashe, I., Man, O., Lancet, D. and Gilad, Y. (2003), 'Different noses for different people', Nat. Genet. Vol. 34, pp. 143-144.

23. Menashe, I., Abaffy, T., Hasin, Y., Goshen, S. et al. (2007), 'Genetic elucidation of human hyperosmia to isovaleric acid', PLoS Biol. Vol. 5, p. e284.

24. Nozawa, M., Kawahara, Y. and Nei, M. (2007), 'Genomic drift and copy number variation of sensory receptor genes in humans', Proc. Natl. Acad. Sci. USA Vol. 104, pp. 20421-20426.

25. Smith, T.D. and Bhatnagar, K.P. (2004), 'Microsmatic primates: reconsidering how and when size matters', Anat. Rec. B New Anat., Vol. 279, pp. 24-31.

26. Gilad, Y., Wiebe, V., Przeworski, M., Lancet, D. et al. (2004), 'Loss of olfactory receptor genes coincides with the acquisition of full trichromatic vision in primates', PLoS Biol. Vol. 2, p. e5.

27. Gilad, Y., Wiebe, V., Przeworski, M., Lancet, D. et al. (2007), 'Correction: Loss of olfactory receptor genes coincides with the acquisition of full trichromatic vision in primates', PLoS Biol. Vol. 5, p. e148.

28. Hiramatsu, C., Melin, A.D., Aureli, F., Schaffner, C.M. et al. (2008), 'Importance of achromatic contrast in short-range fruit foraging of primates', PLoS ONE Vol. 3, p. e3356.

29. Gilad, Y., Man, O., Pääbo, S. and Lancet, D. (2003), 'Human specific loss of olfactory receptor genes', Proc. Natl. Acad. Sci. USA. Vol. 100, pp. 3324-3327.

30. Gilad, Y., Man, O. and Glusman, G. (2005), 'A comparison of the human and chimpanzee olfactory receptor gene repertoires', Genome Res. Vol. 15, pp. 224-230.

31. Kishida, T., Kubota, S., Shirayama, Y. and Fukami, H. (2007), 'The olfactory receptor gene repertoires in secondary-adapted marine vertebrates: Evidence for reduction of the functional proportions in cetaceans', Biol. Lett. Vol. 3, pp. 428-430. 
32. McGowen, M.R., Clark, C. and Gatesy, J. (2008), 'The vestigial olfactory receptor subgenome of odontocete whales: Phylogenetic congruence between gene-tree reconciliation and supermatrix methods', Syst. Biol. Vol. 57, pp. 574-590.

33. Nei, M. and Rooney, A.P. (2005), 'Concerted and birth-and-death evolution of multigene families', Annu. Rev. Genet. Vol. 39, pp. 121-152.

34. Steiger, S.S., Fidler, A.E., Valcu, M. and Kempenaers, B. (2008), 'Avian olfactory receptor gene repertoires: Evidence for a well-developed sense of smell in birds?', Proc. Biol. Sci. Vol. 275, pp. 2309-2317.

35. Osório, J. and Rétaux, S. (2008), 'The lamprey in evolutionary studies', Dev. Genes. Evol. Vol. 218, pp. 221-235.

36. Venkatesh, B., Kirkness, E.F., Loh, Y.H., Halpern, A.L. et al. (2007), 'Survey sequencing and comparative analysis of the elephant shark (Callorhinchus milii) genome', PLoS Biol. Vol. 5, p. e101.

37. Niimura, Y. and Nei, M. (2005), 'Evolutionary dynamics of olfactory receptor genes in fishes and tetrapods', Proc. Natl. Acad. Sci. USA Vol. 102, pp. 6039-6044.

38. Satoh, G. (2005), 'Characterization of novel GPCR gene coding locus in amphioxus genome: Gene structure, expression, and phylogenetic analysis with implications for its involvement in chemoreception', Genesis Vol. 41, pp. 47-57.

39. Delsuc, F, Brinkmann, H., Chourrout, D. and Philippe, H. (2006), 'Tunicates and not cephalochordates are the closest living relatives of vertebrates', Nature Vol. 439, pp. 965-968.

40. Putnam, N.H., Butts, T., Ferrier, D.E., Furlong, R.F. et al. (2008), 'The amphioxus genome and the evolution of the chordate karyotype', Nature Vol. 453, pp. 1064-1071.

41. Raible, F., Tessmar-Raible, K., Arboleda, E., Kaller, T. et al. (2006), 'Opsins and clusters of sensory G-protein-coupled receptors in the sea urchin genome', Dev. Biol. Vol. 300, pp. 461-475.

42. Bargmann, C.I. (2006), 'Comparative chemosensation from receptors to ecology', Nature Vol. 444, pp. 295-301.

43. Vosshall, L.B. and Stocker, R.F. (2007), 'Molecular architecture of smell and taste in Drosophila', Annu. Rev. Neurosci. Vol. 30, pp. 505-533.

44. Benton, R., Sachse, S., Michnick, S.W. and Vosshall, L.B. (2006), 'Atypical membrane topology and heteromeric function of Drosophila odorant receptors in vivo', PLoS Biol. Vol. 4, p. e20.

45. Larsson, M.C., Domingos, A.I., Jones, W.D., Chiappe, M.E. et al. (2004), 'Or83b encodes a broadly expressed odorant receptor essential for Drosophila olfaction', Neuron Vol. 43, pp. 703-714.
46. Sato, K., Pellegrino, M., Nakagawa, T., Nakagawa, T. et al. (2008), 'Insect olfactory receptors are heteromeric ligand-gated ion channels', Nature Vol. 452, pp. 1002-1006.

47. Wicher, D., Schäfer, R., Bauernfeind, R., Stensmyr, M.C. et al. (2008), 'Drosophila odorant receptors are both ligand-gated and cyclic-nucleotideactivated cation channels', Nature Vol. 452, pp. 1007-1111.

48. Robertson, H.A. and Thomas, J.H. (2006), 'The putative chemoreceptor families of C. elegans', Wormbook, ed. The C. elegans Research Community, doi/10.1895/wormbook.1.66.1, http://www.wormbook.org.

49. Cummins, S.F., Erpenbeck, D., Zou, Z., Claudianos, C. et al. (2009), 'Candidate chemoreceptor subfamilies differentially expressed in the chemosensory organs of the mollusc Aplysia', BMC Biol. Vol. 7, 28.

50. Nozawa, M. and Nei, M. (2007), 'Evolutionary dynamics of olfactory receptor genes in Drosophila species', Proc. Natl. Acad. Sci. USA Vol. 104, pp. $7122-7127$.

51. Rivière, S., Challet, L., Fluegge, D., Spehr, M. et al. (2009), 'Formyl peptide receptor-like proteins are a novel family of vomeronasal chemosensors', Nature Vol. 459, pp. 574-577.

52. Liberles, S.D., Horowitz, L.F., Kuang, D., Contos, J.J. et al. (2009), 'Formyl peptide receptors are candidate chemosensory receptors in the vomeronasal organ', Proc. Natl. Acad. Sci. USA Vol. 106, pp. 9842-9847.

53. Hashiguchi, Y. and Nishida, M. (2007), 'Evolution of trace amine associated receptor (TAAR) gene family in vertebrates: Lineage-specific expansions and degradations of a second class of vertebrate chemosensory receptors expressed in the olfactory epithelium', Mol. Biol. Evol. Vol. 24, pp. 2099-2107.

54. Grus, W.E., Shi, P., Zhang, Y.P. and Zhang, J. (2005), 'Dramatic variation of the vomeronasal pheromone receptor gene repertoire among five orders of placental and marsupial mammals', Proc. Natl. Acad. Sci. USA Vol. 102 , pp. 5767-5772.

55. Shi, P. and Zhang, J. (2007), 'Comparative genomic analysis identifies an evolutionary shift of vomeronasal receptor gene repertoires in the vertebrate transition from water to land', Genome Res. Vol. 17, pp. 166-174.

56. Shi, P. and Zhang, J. (2006), 'Contrasting modes of evolution between vertebrate sweet/umami receptor genes and bitter receptor genes', Mol. Biol. Evol. Vol. 23, pp. 292-300.

57. Grus, W.E. and Zhang, J. (2008), 'Distinct evolutionary patterns between chemoreceptors of 2 vertebrate olfactory systems and the differential tuning hypothesis', Mol. Biol. Evol. Vol. 25, pp. 1593-1601.

58. Terakita, A. (2005), 'The opsins', Genome Biol. Vol. 6, 213. 